\title{
Scaling laws in decaying helical hydromagnetic turbulence
}

\author{
Mattias Christensson ${ }^{1}$, Mark Hindmarsh $^{2}$, And Axel BrandenburG ${ }^{3}$ \\ ${ }^{1}$ Operational Research, Strategy and Change Division, ECGD, Harbour Exchange Square, London E14 9GS, UK \\ ${ }^{2}$ Astronomy Centre, University of Sussex, Brighton BN1 9QH, UK \\ ${ }^{3}$ Nordita, Blegdamsvej 17, DK-2100 Copenhagen, Denmark
}

Received 29 April 2005; accepted 20 May 2005; published online 1 July 2005

\begin{abstract}
We study the evolution of growth and decay laws for the magnetic field coherence length $\xi$, energy $E_{\mathrm{M}}$ and magnetic helicity $H$ in freely decaying 3D MHD turbulence. We show that with certain assumptions, self-similarity of the magnetic power spectrum alone implies that $\xi \sim t^{1 / 2}$. This in turn implies that magnetic helicity decays as $H \sim t^{-2 s}$, where $s=\left(\xi_{\text {diff }} / \xi_{H}\right)^{2}$, in terms of $\xi_{\text {diff }}$, the diffusion length scale, and $\xi_{\mathrm{H}}$, a length scale defined from the helicity power spectrum. The relative magnetic helicity remains constant, implying that the magnetic energy decays as $E_{\mathrm{M}} \sim t^{-1 / 2-2 s}$. The parameter $s$ is inversely proportional to the magnetic Reynolds number $R e_{\mathrm{M}}$, which is constant in the self-similar regime.
\end{abstract}

Key words: MHD - turbulence - (cosmology:) early universe

(C)0000 WILEY-VCH Verlag GmbH \& Co. KGaA, Weinheim

\section{Introduction}

Magnetic fields are ubiquitous in the Universe, being observed in objects from planets to galaxy clusters (Zeldovich et al. 1983, Ruzmaikin et al. 1988, Kronberg 1994). In galaxies and galaxy clusters, the typical strength is of order a few $\mu$ Gauss, which is thought to be produced by dynamo action on a seed field. In galaxies the dynamo timescale is roughly a rotation period, $10^{8} \mathrm{yr}$, and a simple calculation (Ruzmaikin et al. 1988) based on the age of a typical galaxy shows that the seed field must have been about $10^{-20}$ Gauss, or perhaps less in the currently favored models with a cosmological term (Davis et al. 1999).

There is no shortage of ideas for generating this seed field. The more conventional astrophysical explanations are based on a Biermann battery operating at the era of reionization (see, e.g., Gnedin et al. 2000, and references therein). There are more speculative ideas based on various generation mechanisms in the early Universe (Grasso \& Rubinstein 2001), which have the common feature of producing stochastic, homogeneous and isotropic magnetic and velocity fields, characterized by their power spectra and initial length scales. Some of these mechanisms produce stochastic fields with non-zero magnetic helicity (Joyce 1997, Cornwall 1997, Vachaspati 2001) the first of these being maximally helical. Another common feature of these generation mechanisms in the early Universe is that they last for a short time only, typically much less than the time it takes for the Universe to dou- ble in size at the time of generation, after which the magnetic fields decay. As we are discussing mechanisms operating at the era of the electroweak phase transition $\left(10^{-11} \mathrm{~s}\right)$ or before, field is generated essentially instantaneously compared with any current astrophysical or cosmological timescale.

The subsequent decay of these primordial fields, and also of those in star-forming regions, motivates the study of freely decaying magnetohydrodynamic (MHD) turbulence (Mac Low et al. 1998, Biskamp \& Müller 1999, Müller \& Biskamp 2000, Christensson et al. 2001). The decay will not in general just be through linear dissipation, as the fields in the early are likely to have high magnetic Reynolds number because of the high conductivity of a fully ionized relativistic plasma (we recall that $R e_{\mathrm{M}}=\xi v / \eta$, where $\xi$ and $v$ are the typical length scale and velocity of the system, and $\eta$ the conductivity). This has been taken to mean in the cosmological context that the magnetic field is frozen into the plasma, and the scale length of the field increases only with the expansion of the Universe. This is in general untrue, because the plasma can move, and turbulence can transfer energy to different length scales (Brandenburg et al. 1996).

Thus a study of decaying MHD turbulence is required in order to calculate quantities such as the size of the seed for the galactic dynamo or the amplitude of the perturbations in the temperature of the Cosmic Microwave Background (CMB) radiation arising from primordial magnetic field generation (Durrer et al. 1998, 2003, Caprini \& Durrer 2001, Caprini et 
al. 2003). Our numerical results (Christensson et al. 2001) uncovered decay laws for magnetic fields which were not those one would expect from purely linear dissipative processes. In particular we saw that helical fields decayed more slowly than non-helical, due to the fact that magnetic helicity is an invariant of ideal MHD. Helicity is known to be important in dynamo theory (Pouquet et al. 1976, Meneguzzi et al. 1981, Brandenburg 2001), and we shall also be able to confirm its importance in decaying turbulence. Our interest here is to try and understand the results, and to compare them with those found by Biskamp \& Müller (1999) and Müller \& Biskamp (2000). In doing so we have developed a new framework for understanding scaling in decaying 3D MHD turbulence, in the case where the fields are close to being maximally helical, as in the mechanisms proposed by Joyce \& Shaposhnikov (1997) and Vachaspati (2001). It should be noted that none of the estimates by Durrer et al. $(1998,2003)$, Caprini \& Durrer (2001), and Caprini et al. (2003) take into account the decay laws we find, and so our results are of direct importance for cosmology.

The decay of the magnetic field in the turbulent case is often presumed to result from an inverse cascade (Pouquet et al. 1976, Meneguzzi et al. 1981, Brandenburg 2001), in which power is transferred locally in $k$-space from small to large scales. However, while it is certainly true that power is transferred from small to large scales, as can been seen from the energy power spectra plotted in Christensson et al. (2001), it was not established that the power is transferred locally, despite the appearance of the term "inverse cascade" in the title of our paper, and so it may be strictly incorrect to call the process a cascade. In fact, a true inverse cascade seems rather unlikely, as large scale power appears almost immediately even from initial conditions which are highly localized in $k$-space. For our analysis in this work it will not matter whether or not there is a cascade, and we will not discuss the matter further. However, we emphasize that the decay of the energy is not simple linear dissipation: there is definitely interesting non-linear physics, as shown by the appreciable Reynolds numbers (100-600) and the processing of the initial power spectra.

Various scaling arguments have been put forward to obtain the growth law for the length scale of the magnetic field and the decay law for the energy. For ideal MHD (infinite conductivity), Olesen (1997) and later Son (1999), Field \& Carroll (2000), and Shiromizu (1998) argued $\xi(t) \sim t^{2 /(n+5)}$ where $t$ is conformal time, and $n$ is the index of the initial magnetic power spectrum. Supporting evidence for this scaling law was also found in two-dimensional MHD simulations (Galtier et al. 1997). Shiromizu's results were based on a renormalization group argument, which has been revisited by Berera \& Hochberg (2001), who do not find evidence for an inverse cascade in the steady state. The effect of having significant helicity was supposed to modify this scaling law to $\xi(t) \sim t^{2 / 3}, E_{\mathrm{M}} \sim t^{-2 / 3}$ (Biskamp 1993, Son 1999, Field \& Carroll 2000). Early numerical experiments with a shell model of the full MHD equations (Brandenburg et al. 1996) suggested $\xi \sim t^{0.25}$, and gave supporting evidence to the inverse cascade. Full MHD simulations by Biskamp \& Müller
(1999) and Müller \& Biskamp (2000) showed, in the helical case, an energy decay law $E_{\mathrm{M}} \sim t^{-1 / 2}$, supported by a phenomenological model, which we will discuss at the end of this work. Decaying non-helical turbulence was studied semianalytically in a shell model by Basu (2000) who found an energy decay law of $E_{\mathrm{M}} \sim t^{-1.2}$. The decay of helical fields was also studied semi-analytically by Sigl (2002), giving a growth law for the length scale between $t^{1 / 2}$ and $t^{2 / 3}$. Unfortunately, direct comparison with our results is otherwise difficult because the correlation functions are expressed in real space. The importance of magnetic helicity in slowing down the decay has been recognized earlier in studies of a low-order model of three-dimensional hydromagnetic flows (Stribling \& Matthaeus 1991), where it was also found that a finite initial cross helicity (not studied in the present paper) can slow down the decay. However, power law exponents of the decay have not been determined for this low-order model.

In an earlier paper (Christensson et al. 2001) we performed 3D simulations both with and without magnetic helicity, starting from homogeneous and isotropic random initial conditions, with power spectra suggested by cosmological applications. We found that the coherence scale of the field grows approximately as $t^{1 / 2}$, with significant transfer of power to small scales in the helical case, which we ascribed to an inverse cascade. The magnetic power spectrum was selfsimilar with an approximately $k^{-2.5}$ behavior at high $k$. We found decay laws for the magnetic and kinetic energies of $t^{-0.7}$ and $t^{-1.1}$ in the helical case, and $t^{-1.1}$ for both in the non-helical case. These are close to, but not identical to those found by Biskamp \& Müller (1999), and we suggested that their relatively large initial length scale, $25 \%$ of the simulation box size, might account for the difference. It should be emphasized that we are interested in the decay only while the scale length of the flows is less than the simulation volume, as we want results relevant to the early Universe where there are no boundaries.

In this paper we present a new theoretical understanding of our numerical results for the power law behavior of the length scale and the energies in the helical case. We show that the key to understanding the power laws is the self-similarity of the magnetic field, coupled with the near-invariance of the helicity, and that the crucial parameter controlling the rate of decay of the magnetic energy and the helicity is the magnetic Reynolds number.

Our theoretical model has analogies with decaying fluid turbulence in 2 dimensions, where there is also an ideal invariant, the kinetic energy, which plays a similar role to the helicity in 3D. Decaying turbulence in 2D (Ting et al. 1986, Chasnov 1997) exhibits self-similarity, and power-law decays in the kinetic energy and enstrophy (mean squared vorticity) are observed in numerical simulations at high Reynolds number (Chasnov 1997). We reserve detailed discussion for Section 5

\section{MHD equations}

The matter and radiation in the early Universe is modeled as an isothermal compressible gas with a magnetic field, which 
is governed by the momentum equation, the continuity equation, and the induction equation, written here in the form (Waleffe 1993)

$$
\begin{aligned}
\frac{\partial \mathbf{u}}{\partial t}=-\mathbf{u} \cdot \nabla \mathbf{u} & -c_{s}^{2} \nabla \ln \rho+\frac{\mathbf{J} \times \mathbf{B}}{\rho} \\
& +\frac{\mu}{\rho}\left(\nabla^{2} \mathbf{u}+\frac{1}{3} \nabla \nabla \cdot \mathbf{u}\right), \\
\frac{\partial \ln \rho}{\partial t} & =-\mathbf{u} \cdot \nabla \ln \rho-\nabla \cdot \mathbf{u}, \\
\frac{\partial \mathbf{A}}{\partial t} & =\mathbf{u} \times \mathbf{B}+\eta \nabla^{2} \mathbf{A}
\end{aligned}
$$

where $\mathbf{B}=\nabla \times \mathbf{A}$ is the magnetic field in terms of the magnetic vector potential $\mathbf{A}, \mathbf{u}$ is the velocity, $\mathbf{J}$ is the current density, $\rho$ is the density, $\mu$ is the dynamical viscosity, and $\eta$ is the magnetic diffusivity. In an expanding Universe the equations are identical when expressed in terms of conformally rescaled fields $\mathbf{B}, \mathbf{u}$ and dissipation parameters $\nu$, and $\eta$. We work in the gauge $A^{0}=-\eta \boldsymbol{\nabla} \cdot \mathbf{A}$ (Subramanian \& Barrow1998, Christensson et al. 2001) under the assumption that $\eta$ is uniform.

In Fourier space it is useful to represent the vector potential in terms of its projection onto an orthogonal basis formed by $\hat{\mathbf{e}}_{+}, \hat{\mathbf{e}}_{-}$and $\hat{\mathbf{k}}$, or

$\mathbf{A}_{\mathbf{k}}=A_{\mathbf{k}}^{+} \hat{\mathbf{e}}_{+}+A_{\mathbf{k}}^{-} \hat{\mathbf{e}}_{-}+A_{\mathbf{k}}^{0} \hat{\mathbf{k}}$.

The two basis vectors $\hat{\mathbf{e}}_{+}$and $\hat{\mathbf{e}}_{-}$can be chosen to be the unit vectors for circular polarization, right-handed and lefthanded respectively. That is $\hat{\mathbf{e}}_{ \pm}=\hat{\mathbf{e}}_{1} \pm i \hat{\mathbf{e}}_{2}$ where $\hat{\mathbf{e}}_{1}$ and $\hat{\mathbf{e}}_{2}$ are unit vectors orthogonal to each other and to $\mathbf{k}$. They are given by $\hat{\mathbf{e}}_{1}=\mathbf{k} \times \hat{\mathbf{z}} /|\mathbf{k} \times \hat{\mathbf{z}}|$ and $\hat{\mathbf{e}}_{2}=\mathbf{k} \times(\mathbf{k} \times \hat{\mathbf{z}}) /|\mathbf{k} \times(\mathbf{k} \times \hat{\mathbf{z}})|$ respectively. $\hat{\mathbf{z}}$ is a reference direction.

Note that since

$i \hat{\mathbf{k}} \times \hat{\mathbf{e}}_{s}=s k \hat{\mathbf{e}}_{s}$

where $s= \pm 1$, this corresponds to an expansion of the magnetic vector potential into helical modes.

Using these basis vectors it is easily seen that the shellaveraged magnetic energy spectrum is

$E_{\mathrm{M}}(k)=2 \pi k^{2}\left\langle\left|\mathbf{B}_{\mathbf{k}}\right|^{2}\right\rangle$

where the amplitude of the magnetic field is given by

$\left|\mathbf{B}_{\mathbf{k}}\right|^{2}=\left(\left|A_{\mathbf{k}}^{+}\right|^{2}+\left|A_{\mathbf{k}}^{-}\right|^{2}\right)|\mathbf{k}|^{2}$

and the expression for the shell-averaged magnetic helicity spectrum $H(k)$ is

$H(k)=4 \pi k^{2}\left\langle\mathbf{A}_{\mathbf{k}}^{*} \cdot \mathbf{B}_{\mathbf{k}}\right\rangle$

where

$\mathbf{A}_{\mathbf{k}}^{*} \cdot \mathbf{B}_{\mathbf{k}}=\left(\left|A_{\mathbf{k}}^{+}\right|^{2}-\left|A_{\mathbf{k}}^{-}\right|^{2}\right)|\mathbf{k}|$.

The function $H(k)$ is bounded in magnitude by the inequality

$|H(k)| \leq 2 k^{-1} E_{\mathrm{M}}(k)$.

A field which saturates the above inequality is maximally helical.

\section{3D MHD simulations of decaying turbulence}

We solve Eqs. (1-3) numerically (Brandenburg 2001) using a variable third order Runge-Kutta timestep and sixth order explicit centered space derivatives. All runs are performed on a $120^{3}$ grid, using periodic boundary conditions, as is appropriate when modeling an infinite volume system. The average density $\langle\rho\rangle=\rho_{0}$ (where the brackets denote a volume average) is conserved.

We use natural units where the speed of light is $c=1$, and fix the unit of length by setting $k_{1}=1$, where $k_{1}$ is the smallest wave number in the simulation box. Hence the box has size $2 \pi$. The scale factor is fixed by setting $\rho_{0}=1$, and $\mathbf{B}$ is measured in units of $\sqrt{\mu_{0} \rho_{0}} c$, where $\mu_{0}$ is the magnetic permeability. We define the mean kinematic viscosity as $\nu \equiv \mu / \rho_{0}$. The sound speed $c_{s}=1 / \sqrt{3}$, as appropriate for a relativistic fluid.

The equations are not quite those for a relativistic gas in the early universe (Brandenburg et al. 1996). However, we have checked that our results change little when using the true relativistic equations in the low velocity limit (Christensson et al. 2001).

We take $\mathbf{u}$ and $\mathbf{B}$ to be homogeneous and isotropic Gaussian random fields drawn from a power-law distribution with a high wavenumber cut-off. The mechanisms for the production of magnetic fields, such as the helical production mechanism of Joyce \& Shaposhnikov (1997), are all stochastic, homogeneous and isotropic, and have an associated length scale $k_{c}^{-1}$. Hence the power spectra, $P_{\mathrm{M}}(k) \equiv\left\langle\mathbf{B}_{\mathbf{k}}^{*} \cdot \mathbf{B}_{\mathbf{k}}\right\rangle$, and $P_{\mathrm{V}}(k) \equiv\left\langle\mathbf{u}_{\mathbf{k}}^{*} \cdot \mathbf{u}_{\mathbf{k}}\right\rangle$ can be initially modeled as

$P_{\mathrm{M}}(k)=A_{M} k^{n_{\mathrm{M}}} e^{-\left(k / k_{c}\right)^{4}}$,
$P_{\mathrm{V}}(k)=A_{V} k^{n_{\mathrm{V}}} e^{-\left(k / k_{c}\right)^{4}}$.

Causality demands that $n_{\mathrm{M}} \geq 2$ and $n_{\mathrm{V}} \geq 0$ (Durrer et al. 1998, 2003, Caprini \& Durrer 2001, Caprini et al. 2003). Note that in the plots it is the shell-integrated energy spectra, $E_{\mathrm{M}, \mathrm{V}}(k)=4 \pi k^{2} \times \frac{1}{2} P_{\mathrm{M}, \mathrm{V}}(k)$, which are shown.

The amplitudes $A_{\mathrm{k}}^{ \pm}$can be chosen independently, provided $A_{-\mathbf{k}}^{* \pm}=A_{\mathrm{k}}^{ \pm}$, which is just the condition that the vector potential be real. Therefore it is possible to adjust the amplitudes $\left|A_{\mathrm{k}}^{+}\right|$and $\left|A_{\mathrm{k}}^{-}\right|$freely and in so doing obtaining a magnetic field with arbitrary magnetic helicity. With our method we are able to put statistically random but maximally helical fields in our initial conditions. In our runs with initial helicity we take $H=H_{\max }$. We took $n_{\mathrm{M}, \mathrm{V}}$ to have the lowest values consistent with causality, and chose $k_{c}=30$.

The initial magnetic energy was taken equal to the kinetic energy, and had the value $5 \times 10^{-3}$ in all runs. The initial density was uniform and equal to 1 . The values for $\nu$ and $\eta$ for the runs presented here are summarized in Table 1

These values were chosen so as to maximize the Reynolds numbers while maintaining numerical stability and resolving the dissipation scales $2 \pi \sqrt{\nu t}$ and $2 \pi \sqrt{\eta t}$. The magnetic dissipation scale is resolved for times $t>1 / N^{2} \eta$, where $N=120$ is the lattice size, with a similar formula for the viscous damping scale. We start taking data at $t \simeq 40$, when $\xi_{\text {diff }} \simeq 2 \pi \sqrt{8}$ for the smallest of our resistivity values, which corresponds to $k \simeq 30$, or approximately 3 lattice points. 


\begin{tabular}{cccccccc}
\hline Run & A & B & C & D & E & F & G \\
\hline $10^{4} \nu$ & 1.0 & 0.7 & 0.7 & 0.7 & 0.7 & 0.7 & 0.7 \\
$10^{4} \eta$ & 1.0 & 0.7 & 0.6 & 0.5 & 0.4 & 0.3 & 0.2 \\
$v t / \xi_{\mathrm{H}}$ & 0.49 & 0.59 & 0.62 & 0.64 & 0.66 & 0.67 & 0.69 \\
$10^{3} R e_{\mathrm{M}}^{-1}$ & 9.2 & 5.4 & 4.5 & 3.7 & 3.0 & 2.4 & 1.6 \\
\hline
\end{tabular}

Table 1. Kinematic viscosity $\nu$, magnetic diffusivity $\eta$, $v t / \xi_{\mathrm{H}}$ and the inverse of the magnetic Reynolds number $R e_{\mathrm{M}}=\xi_{\mathrm{H}} v / \eta$ for our runs. $\xi_{\mathrm{H}}$ is the helicity length scale defined in Eq. (14) and $v$ is the RMS velocity, evaluated at the end of the runs $(t \simeq 80)$.

Examining Fig. 1 of Christensson et al. (2001), we can see the magnetic energy spectrum turning down for $k \gtrsim 30$ at $t=46.3$, showing that the system is exhibiting Ohmic dissipation as required. Under-resolved runs show ringing in real space and the velocity and the magnetic field explode fairly quickly, and so are in practice easy to discard.

\section{Helicity conservation and magnetic energy decay}

In this section we shall take a closer look at the observed scaling laws for helical magnetic fields and see if we can understand them on theoretical grounds. Magnetic helicity is not exactly conserved if $\eta \neq 0$, as it straightforward to show that

$\dot{H}=-2 \eta \int \frac{d^{3} k}{(2 \pi)^{3}} k^{3}\left(\left|A_{\mathbf{k}}^{+}\right|^{2}-\left|A_{\mathbf{k}}^{-}\right|^{2}\right)$.

Hence, providing the power spectrum of the gauge field decays faster than $k^{-6}$ for $k \rightarrow \infty$, implying that the magnetic energy spectrum $E_{\mathrm{M}}(k)$ decays faster than $k^{-2}$, we can define a helicity scale $\xi_{\mathrm{H}}$ such that

$\dot{H}=-8 \pi^{2} \eta H / \xi_{\mathrm{H}}^{2}$.

If we assume that the evolution of $\xi_{\mathrm{H}}$ is described by a power law $\xi_{\mathrm{H}} \sim t^{r}$ it is clear that the solutions to Eq. (14) are qualitatively different depending on the exponent $r$. If and only if $r=1 / 2$ does the magnetic helicity show a power law decay $H \sim t^{-2 s}$

where

$s=\left(\xi_{\text {diff }} / \xi_{H}\right)^{2}$,

in terms of the diffusion scale $\xi_{\text {diff }}=2 \pi \sqrt{\eta t}$. Additional length scales we consider are the integral scale $\xi_{\mathrm{I}}=$ $2 \pi \int d k k^{-1} E_{\mathrm{M}}(k) / \int d k E_{\mathrm{M}}(k)$, the relative helicity scale $\xi_{\mathrm{R}}=\pi|H| / E_{\mathrm{M}}$ and the magnetic Taylor microscale $\xi_{\mathrm{T}}=$ $2 \pi B_{\mathrm{rms}} / J_{\mathrm{rms}}$, where $B_{\mathrm{rms}}$ and $J_{\mathrm{rms}}$ are the RMS magnetic field and current density respectively. It is plausible that all these scales are proportionally related and Fig. 11 shows that this is indeed the case. A theoretical reason for this behavior is given below.

The magnetic helicity is bounded in magnitude by the inequality Eq. (10). An equivalent way to express this is by

$H_{\mathrm{REL}} \equiv \frac{\pi\left|H_{\mathrm{M}}\right|}{\xi_{\mathrm{I}} E_{\mathrm{M}}} \leq 1$,

where $H_{\mathrm{REL}}$ is the relative magnetic helicity. So if the above bound remains approximately saturated, i.e. $H_{\mathrm{REL}} \sim 1$, and

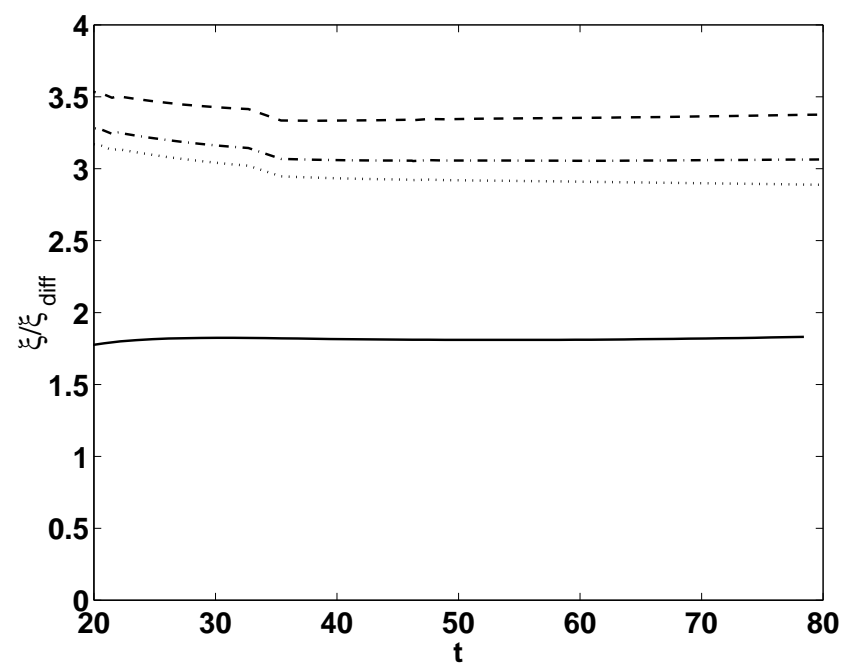

Fig. 1. Time evolution of the ratio of length scale to the diffusion scale for run $\mathrm{D}$. The notation is $\xi_{\mathrm{I}} / \xi_{\mathrm{diff}}$ (dashed), $\xi_{\mathrm{H}} / \xi_{\text {diff }}\left(\right.$ dot-dashed), $\xi_{\mathrm{R}} / \xi_{\text {diff }}$ (dotted) and $\xi_{\mathrm{T}} / \xi_{\text {diff }}$ (continuous). The length scales are defined in Eq. (14) and the subsequent text.

the helical length scale goes as $\xi_{\mathrm{H}} \sim t^{1 / 2}$, the decay law for the magnetic energy is

$E_{\mathrm{M}} \sim t^{-1 / 2-2 s}$.

In any case, the energy cannot decay faster than this. Given $H_{\mathrm{REL}}=\xi_{\mathrm{R}} / \xi_{\mathrm{I}}$, it is seen from Fig. 11 that $H_{\mathrm{REL}}$ is indeed of order unity and does not decay markedly with time.

To characterize the decay laws we define the exponents

$Q(t)=-t \dot{E}_{\mathrm{M}} / E_{\mathrm{M}}, \quad R(t)=-t \dot{H} / 2 H$.

In Fig. 2 we have plotted $R(t)$ versus the quantity $s(t)=$ $\left(\xi_{\text {diff }} / \xi_{H}\right)^{2}$ for several runs with different initial conditions. The time span is $t \sim 40$ to $\sim 80$, i.e. the last half of the simulations. This figure tells us several things. Firstly, it shows us that the value of $R$ is approximately independent of time which confirms the power law decay of $H$. Secondly, Fig. 2 indicates that the quantity $s$ is also approximately independent of time, hence reinforcing the relation $\xi_{H} \sim t^{1 / 2}$. Thirdly, it seems that $R$ and $s$ are almost equal, and not just proportional.

Taking the relative helicity to be constant, it follows from the power-law behavior of $H$ that the energy decay law is indeed $E_{\mathrm{M}} \sim t^{-1 / 2-2 s}$. In the limit of exact conservation of magnetic helicity, $s \rightarrow 0$, the magnetic energy must decay as $E_{\mathrm{M}} \sim t^{-1 / 2}$.

Regarding the physical significance of the parameter $s$ we note that if $\xi_{\mathrm{H}} \simeq v t$, where $v$ is the RMS velocity, (i.e. if the eddy turn-over time is $t$ ) then $s \simeq(2 \pi)^{2} / R e_{\mathrm{M}}$, where $R e_{\mathrm{M}}$ is the magnetic Reynolds number evaluated using the helicity scale $\xi_{\mathrm{H}}$. We have measured $f=v t / \xi_{\mathrm{H}}$ and $R e_{\mathrm{M}}^{-1}$ for all runs, and find that they both change by less than about $10 \%$ between $t=20$ and the end of the runs at $t=80$, giving the final values in Table 1 One can see that there is a linear relation $f=f_{0}+f_{1} / R e_{\mathrm{M}}$ between the two, and a least squares fit gives $f_{0}=0.734 \pm 0.002, f_{1}=-26.6 \pm 0.4$. 


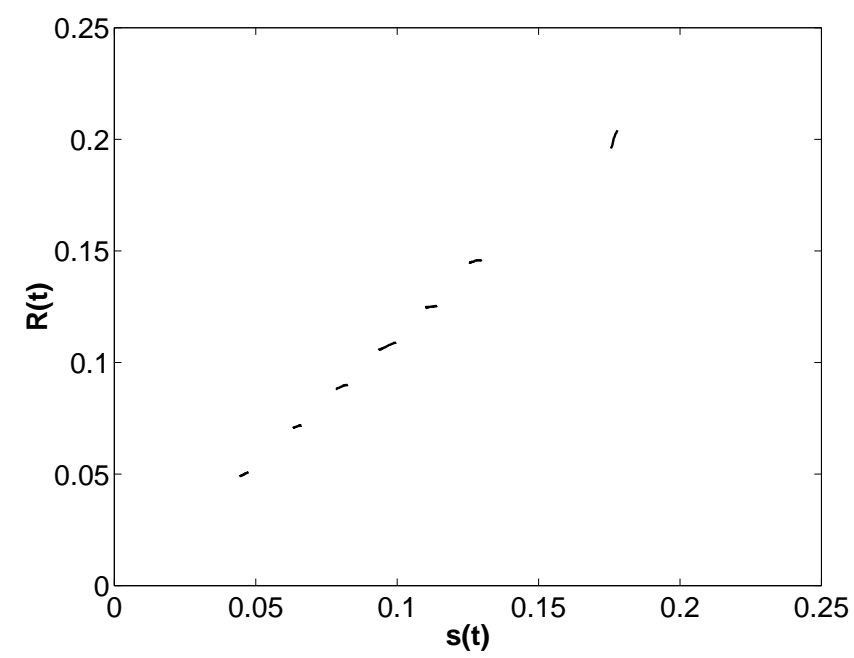

Fig. 2. The quantity $R(t)$, defined by Eq. (19) versus the quantity $s(t)=\left(\xi_{\text {diff }} / \xi_{H}\right)^{2}$ for runs listed in Table 11 The length scales $\xi_{H}$ and $\xi_{\text {diff }}$ are defined in Eq. (14) and the subsequent text. The time span is approximately from $t \simeq 40$ to $\simeq 80$, i.e. the last half of the simulations.

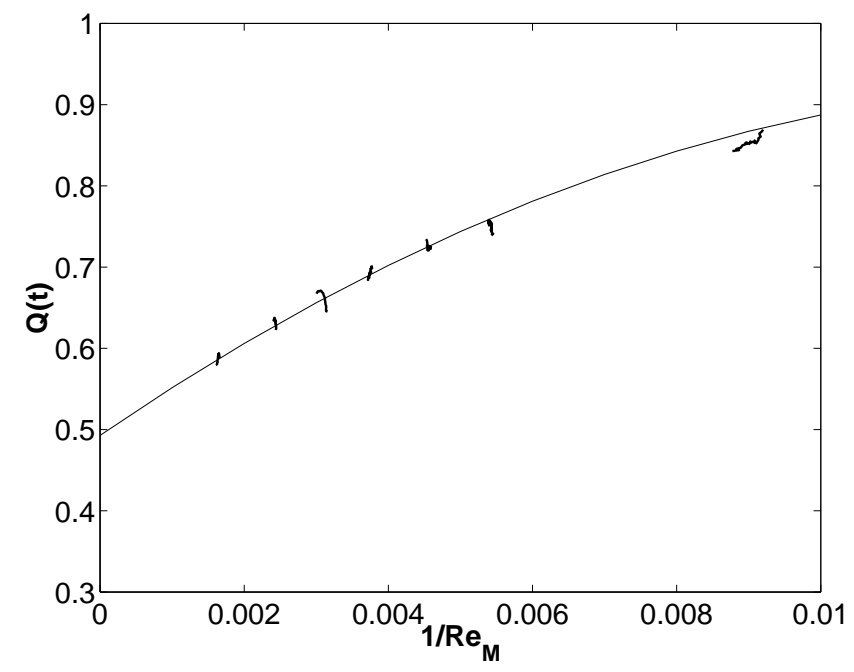

Fig. 3. Energy decay exponent $Q$, defined in Eq. 19, plotted against the inverse magnetic Reynolds number $R e_{\mathrm{M}}^{-1}$, calculated using the helicity scale $\xi_{\mathrm{H}}$, for the last half of the runs listed in Table 1 The fit is a quadratic least squares fit using the final values for each run.

The linear relation between $f$ and $R e_{\mathrm{M}}^{-1}$ implies that there should be a linear relation between $s$ and $R e_{\mathrm{M}}^{-1}$, and hence a quadratic relation between $Q(t)$, the energy decay exponent defined in Eq. [19, and $R e_{\mathrm{M}}^{-1}$. Fig. 3] showing $Q$ and $R e_{\mathrm{M}}^{-1}$ for $40<t<80$, confirms that this is indeed the case, with asymptote at large $R e_{\mathrm{M}}$ consistent with $Q=1 / 2$. Furthermore, it also shows that both $Q$ and $R e_{\mathrm{M}}^{-1}$ change little over the last half of the runs.

Let us now turn to the decay of magnetic energy which we use to show that the characteristic length scale of the field $\xi$ must scale as $t^{1 / 2}$, assuming the scaling form of the magnetic energy power spectrum

$E_{M}(k)=\xi^{-q} g_{M}(k \xi)$

found in Christensson et al. (2001). From the MHD equations one can show

$\dot{E}_{M}=-\int d^{3} x \mathbf{u} \cdot(\mathbf{J} \times \mathbf{B})-\eta \int d^{3} x \mathbf{J}^{2}$.

We assume that the dissipation term, if not dominant, always contributes a constant fraction to the energy loss. If $E_{\mathrm{M}}(k) \sim$ $k^{-z}$ at high $k$, with $z<3$, then the integral is dominated by the high- $k$ cut-off $\xi_{\text {diff }}^{-1}$. We do indeed see a power spectrum with $2<z<3$ (Christensson et al. 2001), which means that there is a well-defined helicity decay law, and that the energy decay is dominated by the smallest scales. Hence the dissipative loss $\epsilon_{M}=-\dot{E}_{M}$ is then

$\epsilon_{M} \sim \xi^{-q-z} \xi_{\text {diff }}^{-3+z}$.

However, on integrating the energy power spectrum and then differentiating with respect to time,

$\epsilon_{M} \sim \dot{\xi} \xi^{-q-2} \sim t^{-1} \xi^{-q-1}$.

If we use the fact that $\xi_{\text {diff }} \sim t^{1 / 2}$, we find on equating the $\epsilon_{M}$ found in the two different ways, $\xi^{-q-z} \sim t^{-(1+z) / 2} \xi^{-q-1}$, or

$\xi \sim t^{1 / 2}$.

In deriving the power law for the length scale of the magnetic field, it is not necessary that the dissipative terms account for all the energy loss, just that it scales the same way as the energy loss and hence contributes a fixed fraction to the total.

From the scaling form of the energy power spectrum 20 , the energy decay law 18 and the length scale growth law 24. one can easily show that a causal power spectrum with $n=2$ behaves as

$E_{M}(k, t) \sim k^{4} t^{2-2 s}$

at low $k$. This growth at large scales should be taken into account when calculating the strength of primordially-produced fields, and will weaken the strong bounds derived by Durrer et al. (1998, 2003), Caprini \& Durrer (2001), Caprini et al. (2003).

\section{Comparison with 2D fluid turbulence}

There is an important similarity between 3D MHD and 2D fluid turbulence: both exhibit inverse cascade behavior associated with the existence of ideal invariants (Biskamp 1993) In 2D fluid turbulence the energy shows an inverse cascade (Frisch \& Sulem 1984), which is similar to the inverse cascade of magnetic helicity in 3D MHD turbulence. There are also some corresponding similarities between the decay laws in decaying MHD turbulence and decaying 2D fluid turbulence. Chasnov (1997) studied 2D velocity fields initialized with a Gaussian distribution, whose energy spectrum $E(k)$ is a power law at low $k$ and has an exponential cut-off at high $k$, much as we do. 
From the Navier-Stokes equation for an incompressible fluid one can easily show

$$
\frac{\partial\left\langle\mathbf{u}^{2}\right\rangle}{\partial t}=-2 \nu\left\langle\boldsymbol{\omega}^{2}\right\rangle, \quad \frac{\partial\left\langle\boldsymbol{\omega}^{2}\right\rangle}{\partial t}=-2 \nu\left\langle\nabla \boldsymbol{\omega}^{2}\right\rangle,
$$

where $\boldsymbol{\omega}=\boldsymbol{\nabla} \times \mathbf{u}$ is the vorticity (which is a scalar in two dimensions, i.e. $\boldsymbol{\omega}=\omega \hat{\mathbf{z}})$. We define two length scales from the enstrophy, $\left\langle\boldsymbol{\omega}^{2}\right\rangle / 2$, and the palinstrophy, $\left\langle\nabla \boldsymbol{\omega}^{2}\right\rangle / 2$,

$l^{2}=\left\langle\mathbf{u}^{2}\right\rangle /\left\langle\boldsymbol{\omega}^{2}\right\rangle, \quad l_{\mathrm{p}}^{2}=\left\langle\boldsymbol{\omega}^{2}\right\rangle /\left\langle\nabla \boldsymbol{\omega}^{2}\right\rangle$,

in addition to the diffusion length scale $l_{\text {diff }}=\sqrt{\nu t}$. Postulating power-law decays for the energy and enstrophy,

$\left\langle\mathbf{u}^{2}\right\rangle \sim t^{n}, \quad\left\langle\boldsymbol{\omega}^{2}\right\rangle \sim t^{m}$,

we find that $m=n-1$, and that

$n=-2 \nu t\left\langle\boldsymbol{\omega}^{2}\right\rangle /\left\langle\mathbf{u}^{2}\right\rangle=-2\left(l_{\text {diff }} / l\right)^{2}$.

Hence, a power-law decay again requires that $l \sim t^{1 / 2}$, and in the limit that the length scale of the flow becomes large compared with the diffusion scale, the energy is conserved. This is analogous to the conservation of magnetic helicity in 3D MHD.

However, the Reynolds number, approximately constant in our simulations, behaves rather differently in 2D fluid turbulence. Defining $R e=u l / \nu$, with $u=\left\langle\mathbf{u}^{2}\right\rangle^{1 / 2}$, we find $R e \sim t^{(1+2) / 2}$, at least for large Reynolds numbers. For small Reynolds numbers we expect $R e$ to decay to zero through dissipation, pointing to the existence of a critical Reynolds number $R e_{c}$, at which the system can remain for long periods, if initialized close enough to the correct value (Chasnov 1997).

The energy spectrum of decaying turbulence is also found to be self-similar in 2D. Similar arguments to those presented in Section 4 show that self-similarity again implies $l \sim t^{1 / 2}$. These arguments also show that if the palinstrophy length scale $l_{\mathrm{p}}$ is of the same order of magnitude as the enstrophy length scale $l$, and that enstrophy is being dissipated at the diffusion scale $l_{\text {diff }}$, the high- $k$ exponent of a power-law energy spectrum must be between -4 and -5 . Intriguingly, the results of Chasnov (1997) seem to indicate a power law somewhat steeper than the $k^{-3}$ behavior expected in stationary turbulence Kraichnan \& Nagarajan 1967, Batchelor 1969.

\section{Discussion and conclusions}

We have studied the evolution of decaying 3D MHD turbulence involving maximally helical magnetic fields. For finite magnetic diffusivity there emerges an important quantity $s=\left(\xi_{\text {diff }} / \xi_{\mathrm{H}}\right)^{2}$, where $\xi_{\mathrm{H}}$ is the helicity scale defined in Eq. 14), and $\xi_{\text {diff }}$ is the diffusion scale. We find $\xi_{\mathrm{H}} \simeq v t$, where $v$ is the RMS velocity, and hence that $s \propto R e_{\mathrm{M}}^{-1}$, the magnetic Reynolds number evaluated using the helicity scale. The magnetic field coherence length (which can be equally well expressed as the integral, helicity or relative helicity scales) goes as $\xi \sim t^{1 / 2}$, magnetic helicity $H_{\mathrm{M}} \sim t^{-2 s}$ and magnetic energy $E_{\mathrm{M}} \sim t^{-1 / 2-2 s}$. A corollary is that $R e_{\mathrm{M}}$ is constant once the system has reached self-similarity. Furthermore, we can extrapolate to the limit of very large magnetic Reynolds numbers, useful for example in the early Universe, to find $H$ constant and $E_{\mathrm{M}} \sim t^{-1 / 2}$.

Our model for the scaling laws should be compared with that of Biskamp \& Müller (1999). The first difference is that they assumed that the non-linear term in the evolution equation for the magnetic field was the dominant source of energy loss for the magnetic field, and that the magnetic field was asymptotically the dominant contributor to the total energy $E$, expressed as $\Gamma \equiv E_{\mathrm{V}} / E_{\mathrm{M}} \ll 1$. Then we can write

$\dot{E} \sim \Gamma^{\frac{1}{2}} E^{3 / 2} / \xi$,

where $\xi$ is a length scale of the magnetic field. They then found the phenomenological relation $\Gamma \simeq E / H$, which, when coupled with $E \simeq H / \xi$ and the conservation of $H$, gives $E \sim t^{-1 / 2}$.

We emphasize that this model is not inconsistent with ours. We infer $E_{\mathrm{V}} \sim t^{-1}$ from the relation $\xi_{\mathrm{H}} \simeq v t$, and hence that $\Gamma \sim t^{-1 / 2+2 s} \sim(E / H) t^{2 s}$. The difference between Biskamp \& Müller's assumed relation $\Gamma \sim E / H$ and ours is small at large magnetic Reynolds numbers where $s \rightarrow 0$. Furthermore, both approaches need to assume only that the non-linear and dissipative terms in Eq. 21) are not sub-dominant (rather than dominant) and it turns out that both scale with time in the same way, as $E_{\mathrm{M}} / t$. In our simulations dissipation typically accounted for about $60 \%$ of the energy loss in the period $t \simeq 40$ to $t \simeq 80$, which means that the field is not force-free.

We believe that our model has certain advantages, in that the assumptions going into it give more physical insights than the phenomenological (and dimensionally incomplete) relation $\Gamma \sim E / H$. Our assumptions are that the magnetic power spectrum exhibits a self-similar form (20), with power-law behavior $k^{-z}$ at high $k$, that resistive dissipation occurs predominantly at the diffusion scale $\xi_{\text {diff }}$, that there is a separate helicity scale $\xi_{\mathrm{H}}$, from which it follows that $2<z<3$. We also assume that the eddy turn-over time $\xi / v$ is $t$, and that the relative helicity is asymptotically constant, i.e. that the energy decays as fast as possible as is consistent with helicity conservation. Given these assumptions, it follows that the dissipative and non-linear terms in the magnetic energy loss equation (21) scale the same way, and we can infer that $\xi \sim t^{1 / 2}$. From this we derive a scaling law for $H$, finding as expected that $H$ is conserved in the limit of large magnetic Reynolds number, and with the assumption of constant relative helicity, we obtain that $E \sim t^{-1 / 2}$ in the same limit.

In summary, for both 3D helical MHD and 2D fluids, the key to understanding the free decay of turbulence is selfsimilarity, coupled to a separation between the scale of the flow and the diffusion scale.

Note added. Since the first draft of this work appeared as astro-ph/0209119,v1, there have been several developments. A new review of magnetic fields in cosmology has appeared (Giovannini 2004). Using different scaling arguments, Campanelli (2004) has rederived our scaling laws for decaying helical turbulence, and extended the analysis to the non-helical case, where the ideal limit has $E_{\mathrm{M}} \propto t^{-1}, E_{\mathrm{V}} \propto t^{-1}$, and $\xi \propto t^{1 / 2}$. Extensive 3D numerical simulations, including the interesting case of Prandtl number larger than unity, have 
been carried out by Banerjee \& Jedamzik (2003, 2004). The authors did not seem to be aware of our work or of Campanelli (2004) and did not attempt to analyse the decay of the helicity, kinetic or magnetic energies in its light. However, examination of their Figs. 1 and 7 in the later paper show decay laws close to $t^{-0.5}$ and $t^{-1}$ for the helical and non-helical cases respectively (with Prandtl number unity). They do not quote Reynolds numbers for the simulations so an exact comparison cannot be made. There has also been further work on effects of primordial fields on the CMB (Kosowsky et al. 2005, Kahniashvili \& Kahniashvili 2005) and on the evolution of magnetic fields in the post-recombination era (Sethi \& Subramanian 2004).

Acknowledgements. This work was conducted on the Cray T3E and SGI Origin platforms using COSMOS Consortium facilities, funded by HEFCE, PPARC and SGI. We also acknowledge computing support from the Sussex High Performance Computing Initiative. MH thanks for NORDITA for hospitality, and MC the Astronomy Centre at the University of Sussex.

\section{References}

Banerjee R., Jedamzik, K. 2003, PhRvL, 91, 252301 [Erratum-ibid. 2004, PhRvL, 93, 179901]

Banerjee, R., Jedamzik, K. 2004, PhRvD, 70, 123003

Basu, A. 2000, PhRvE, 61, 1407

Batchelor, G. K. 1969, PhFluS, II 12, 233

Berera, A., Hochberg, D.: 2001, arXiv:cond-mat/0103447

Biskamp, D. 1993, Nonlinear magnetohydrodynamics (Cambridge University Press, Cambridge)

Biskamp, D., Müller, W.-C. 1999, PhRvL, 83, 2195

Brandenburg, A. 2001, ApJ, 550, 824

Brandenburg, A., Enqvist, K., Olesen, P. 1996, PhRvD, 54, 1291

Campanelli, L. 2004, PhRvD, 70, 083009

Caprini, C., Durrer, R. 2001, PhRvD, 65, 023517

Caprini, C., Durrer, R., Kahniashvili, T. 2004, PhRvD, 69, 063006

Chasnov, J. R. 1997, PhLB, 9, 171

Christensson, M. 2002, Thesis, Univ. Sussex (Cosmological magnetic fields in the early universe)

Christensson, M., Hindmarsh, M., Brandenburg, A. 2001, PhRvE, 64,056405

Cornwall, J. M. 1997, PhRvD, 56, 6146

Davis, A. C., Lilley, M., Tornkvist, O. 1999, PhRvD, 60, 021301

Durrer, R., Caprini, C. 2003, JCAP, 0311, 010

Durrer, R., Kahniashvili, T., Yates, A. 1998, PhRvD, 58, 123004

Field, G. B., Carroll, S. M. 2000, PhRvD, 62, 103008

Frisch, U., Sulem, P. L. 1984, PhLB, 27, 1921

Galtier, S., Politano, H., Pouquet, A. 1997, PhRvL, 79, 2807

Giovannini, M. 2004, Int. J. Mod. Phys. D, 12, 391

Gnedin, N. Y., Ferrara, A., Zweibel, E. G. 2000, ApJ, 539, 505

Grasso, D., Rubinstein, H. R. 2001, PhR, 348, 163

Joyce, M., Shaposhnikov, M. 1997, PhRvL, 79, 1193

Kahniashvili, T., Ratra, B., arXiv:astro-ph/0503709

Kosowsky, A., Kahniashvili, T., Lavrelashvili G., Ratra, B. 2005, PhRvD, 71, 043006

Kraichnan, R. H., Nagarajan, S. 1967, PhLB, 10, 859

Kronberg, P. P. 1994, Rep. Prog. Phys., 57, 325

Mac Low, M.-M., Klessen, R. S., Burkert, A. 1998, PhRvL, 80, 2754

Meneguzzi, M., Frisch, U., Pouquet, A. 1981, PhRvL, 47, 1060

Müller, W.-C., Biskamp, D. 2000, PhRvL, 84, 475

Olesen, P. 1997, PhLB, 398, 321

Pouquet, A., Frisch, U., Léorat, J. 1976, JFM, 77, 321

Ruzmaikin, A. A., Sokoloff, D. D. Shukurov, A. M. 1988, Magnetic fields of galaxies (Kluwer, Dordrecht)
Sethi, S. K., Subramanian, K. 2005, MNRAS, 356, 778

Shiromizu, T. 1998, PhLB, 443, 127

Sigl, G. 2002, PhRvD, 61, 123002

Son, D. T. 1999, PhRvD, 59, 063008

Stribling, T., Matthaeus, W. H. 1991, PhLB, B 3, 1848

Subramanian, K., Barrow, J. D. 1998, PhRvD, 58, 083502

Ting, A. C., Montgomery, D., Matthaeus, W. H. 1986, PhLB, 29, 3261

Vachaspati, T. 2001, PhRvL, 87, 251302

Waleffe, F. 1993, PhLB, A5, 677

Zeldovich, Ya. B., Ruzmaikin, A. A., Sokoloff, D. D. 1983, Magnetic fields in astrophysics (Gordon \& Breach, New York) 